\title{
Measuring sub-mm structural displacements using QDaedalus: a digital clip-on measuring system developed for Total Stations
}

\author{
E. Charalampous ${ }^{1}$, P. Psimoulis ${ }^{1,2^{*}}$, S. Guillaume ${ }^{1}$, M. Spiridonakos ${ }^{3}$, R. Klis ${ }^{3}$, B. Bürki ${ }^{1}$, M. \\ Rothacher $^{1}$, E. Chatzi ${ }^{3}$, R. Luchsinger ${ }^{4}$, G. Feltrin ${ }^{4}$ \\ ${ }^{1}$ Geodesy \& Geodynamics Lab., Institute of Geodesy and Photogrammetry, Dept. of Civil \\ Engineering, ETH Zurich, Switzerland \\ ${ }^{2}$ Nottingham Geospatial Institute, The University of Nottingham, Nottingham, UK \\ ${ }^{3}$ Institute of Structural Engineering, Dept. of Civil Engineering, ETH Zurich, Switzerland \\ ${ }^{4}$ Swiss Federal Laboratory for Materials Science and Technology (EMPA), Zurich, Switzerland
}

\begin{abstract}
The monitoring of rigid structures of modal frequencies greater than $5 \mathrm{~Hz}$ and sub-mm displacement is mainly based so far on relative quantities from accelerometers, strain gauges etc. Additionally geodetic techniques such as GPS and Robotic Total Stations (RTS) are constrained by their low accuracy (few mm) and their low sampling rates. In this study the application of QDaedalus is presented, which constitutes a measuring system developed at the Geodesy and Geodynamics Lab, ETH Zurich and consists of a small CCD camera and Total Station, for the monitoring of the oscillations of a rigid structure. In collaboration with the Institute of Structural Engineering of ETH Zurich and EMPA, the QDaedalus system was used for monitoring of the sub-mm displacement of a rigid prototype beam and the estimation of its modal frequencies up to $30 \mathrm{~Hz}$. The results of the QDaedalus data analysis were compared to those of accelerometers and proved to hold sufficient accuracy and suitably supplementing the existing monitoring techniques.
\end{abstract}

Keywords: CCD camera, total station, sub-mm oscillation, high modal frequency

*Corresponding author, Lecturer in Geospatial Eng, E-mail: Panagiotis.Psimoulis@nottingham.ac.uk 


\section{Introduction}

There has been a broad interest during the last years for the displacement measurement of oscillations and deflections of various engineering structures in response to dynamic load, such as wind, traffic load (Roberts et al., 2004; Psimoulis and Stiros, 2007; Chatzi and Fuggini, 2012) and quasi-static loads corresponding mainly to temperature effects (Behr and Hudnut, 1998; Koo et al., 2012). The measuring of the displacement of structures deflection aims mainly to the monitoring of the structural health by identifying changes in the structural characteristics (displacement, modal frequencies, etc.; Spiridonakos and Fassois, 2009; Chatzi and Smyth, 2013) due to aging, earthquake damage or postdamage repairs (Hamsphire and Adeli, 2000) and to eventually use them as input in structural design (Bardakis and Fardis, 2011).

Mainly throughout the last decade GPS and Robotic Total Stations are broadly used for the monitoring of displacement of structures, focusing mainly on bridges (Meng et al., 2007; Stiros and Psimoulis, 2012; Psimoulis and Stiros, 2013; Moschas et al., 2013, Moschas and Stiros, 2014), tall buildings and towers (Breuer et al., 2008; Li et al., 2006). Even though, the improvement and the development of GPS (i.e. high-rate receivers), and RTS (mm-accuracy, Psimoulis and Stiros, 2008; Zarikas et al., 2010; Psimoulis and Stiros, 2013) technology in combination with advanced data processing methodologies (Spiridonakos and Fassois, 2009; Psimoulis and Stiros, 2012; Chatzi and Smyth, 2013) have broadened the application field in monitoring structures with vibration frequency up to $4-5 \mathrm{~Hz}$ and a few $\mathrm{mm}$ of displacement, there are still limitations in monitoring displacement of sub-mm amplitude and frequency greater than $5 \mathrm{~Hz}$.

Techniques have been developed for the monitoring of high-rate dynamic sub-mm displacement of structures using interferometers (Gentile and Bernardini, 2010; Gentile, 2010), which though provide the axial displacement of a structure. Image-processing techniques are also implemented for the monitoring of sub-mm static displacements, such as concrete cracks (Niemeier et al., 2008), or even the dynamic deformation of structures, with main disadvantages being until recently the complexity of the techniques (Olaszek, 1999), the high-cost of the devices (Wahbeh et al., 2003) and the limitation of 2-D displacement monitoring (Fukuda et al., 2010). However, the image/video-recording techniques have been adopted and developed by many manufacturers (i.e. GSI VSTARS, AICON, GOM, etc.), focusing mainly in industrial applications by providing even the 3D dynamic deformation of rigid structures, with sub-mm accuracy and very high frame rates $(>1000 \mathrm{~Hz}$; Luhnmann, 2010; Kim and 
Chen, 2012). A similar attempt has been lately made by Geodesy and Geodynamis Lab of ETH Zurich, for the development of a 3D monitoring technique, known as QDaedalus,

More specifically, QDaedalus system was initially designed and developed for the automated on line astro-geodetic measurements. However, its potential for application in deformation and vibration monitoring of structures with sub-mm response was quickly revealed. In this study, the accuracy and the reliability of QDaedalus is assessed in the monitoring of sub-mm displacement of a rigid structural system. The evaluation of QDaedalus was based on experiments which were conducted in collaboration with the Institute of Structural Engineering and Swiss Federal Laboratories for Materials Science and Technology (EMPA), both parts of ETH, focusing on the monitoring of the response of a prototype pump-pressure beam, technology known as "Tensairity". The aim of the experiments was the estimation of the modal frequencies of the beam and the detection of changes of the structure stiffness due to the different air-pressure level of the beam, based on the QDaedalus measurements. The accuracy of the estimation of the modal frequencies using the QDaedalus measurements, is assessed by comparing them with the estimated modal frequencies using the accelerometers measurements. In this study the first results of the QDaedalus monitoring campaign are presented and discussed.

\section{QDaedalus System}

\subsection{Implementation}

The initial aim of QDaedalus, focusing on astro-geodetic measurements, led to the main idea of replacing the human vision in a non-invasive way by a CCD camera mounted on a Total Station, in order to avoid influences from the observer's subjective perception. The potential of CCD camera to trigger and handle the exposures and the corresponding epochs with adequate accuracy in combination with the accuracy of the total station telescope, can provide highly refined digital angular measurements.

Following the main concept, a monochrome CCD camera from Allied Vision Technologies (AVT; Guppy F-080C CCD camera) was chosen, whose shutter can be triggered by pulses of an external software. The resolution of the CCD camera is $1024 \times 768$ pixels with a size of $4.65 \times 4.65$ microns and a capable of 60 frames per second. However, the latter can increase by reducing the size of the captured image, in case of very high frequency motion. The CCD camera is mounted at the telescope, by using 
an adaptor, in order to replace the eyepiece. For measurements of distance longer than $\sim 10 \mathrm{~m}$, an additional pluggable front lens is used in order to shift slightly the focal length from the plane of telescope reticle to the plane of the CCD camera.

The second main characteristic of QDaedalus is the software for performing Optical Target Recognition (OTR), implemented for the detection of the monitoring target(s) and for their image processing. From the large spectrum of the existing image processing algorithms, three algorithms were selected as the most appropriate for the applications of QDaedalus (Burki et al., 2010): i) center of mass used mainly for monitoring of actively illuminated targets such as stars, diodes, lamps, etc., ii) template least squares matching, appropriate for very accurate calibration when it is needed and iii) circle matching, usually useful for industrial applications (measuring spheres, etc.).

Based on these techniques, the application field of QDaedalus is broadened, apart from the astrogeodetic measurements, in automated terrestrial surveying, deformation monitoring, microtriangulations application and photographic documentation. According to the application of QDaedalus the appropriate setup is differentiated. The additional parts of QDaedalus are: i) a GPS receiver, which can provide the captured images in GPS time, important for applications where accurate timing is needed, iii) a triggering device with 8 specific pulses modes (with frequencies following the power of two sequence) for the synchronization and triggering of several QDaedalus systems and iv) a supplementary software for image steering, from the CCD camera to the laptop. More detailed information for the functional characteristics and the OTR algorithms of QDaedalus can be found in Bürki et al. (2010).

\subsection{Application in Structural Monitoring}

The application of the QDaedalus in structural monitoring is based on three of its main aspects: i) the OTR technique ii) the triggered images and iii) the use of Total Station. More specifically, LEDs are set as targets at the monitoring points of the structure, and their position is captured by the images of the CCD camera. By employing the OTR technique the position of each LED is defined in every captured image and the corresponding position is given in pixels $p_{x}, p_{z}$, according to an arbitrary 2-D Cartesian coordinate system, where the origin is placed on the top left corner of the image (Fig.1a). The Cartesian coordinate system is common for all the images as the QDaedalus system is not moved during the monitoring. Based on the approximate distance $d$ between QDaedalus and the LED-target 
and the precisely defined angular field $\theta$ of the total station, which is fixed that for each pixel is equal to 4 arcsec, each pixel can be transformed into length units based on the equation:

$$
\left(\begin{array}{l}
x \\
z
\end{array}\right)=-d \cdot \sin \theta \cdot\left(\begin{array}{l}
p_{x} \\
p_{z}
\end{array}\right)
$$

where $x$ and $z$ are the Cartesian coordinates of the corresponding LED, in meters in the transformed 2D Cartesian Coordinate System of the image. The displacement of the monitored LED is derived by comparing the position (in length units) of the corresponding LED in the successive captured images to the common 2-D Cartesian Coordinate System of the images.

The triggering device defines precisely the sampling rate of the captured images, which leads to the precise time history of the displacement, resulting finally to the displacement time series of the LEDtargets in the 2-D Cartesian coordinate system. Also, the connection of QDaedalus with the GPS receiver, provides the displacement time series in GPS time.

Furthermore, two or more QDaedalus can be combined and synchronised by the triggering device, for monitoring the same LED-targets from different control points, providing the 3-D displacement of the targets (Fig. 1b). Based on the local Cartesian coordinate systems of each QDaedalus system $\left(\mathrm{x}_{1}, \mathrm{z}_{1}\right.$ and $\mathrm{x}_{2} \mathrm{z}_{2}$ ) and their position relative to each other and the target(s), the 3-D displacement of the target(s) can be computed in an independent Cartesian coordinate system $\left(\mathrm{x}_{\mathrm{g}} \mathrm{yg}_{\mathrm{g}} \mathrm{z}_{\mathrm{g}}\right)$. Additional advantage of using Total Station is the fast deployment of the network for the QDaedalus systems, the accurate setting-out of the control points and the easy orientation of the QDaedalus systems according to the surveying network, which makes possible also the monitoring of the absolute positioning of a target and not only its relative displacement; the latter is rather important for slow, static displacements of structures (Behr and Hudnut, 1998; Koo et al., 2012).

Finally one of the main advantages of QDaedalus, which is not common in image-recording techniques, is that calibration is not needed before the measurements, apart from on-site modifications of the image saturation, brightness, etc. which may be needed for capturing more sharp and clear images. The only requirement, which is needed is the measurement of the distance $d$ of the QDaedalus from the monitored LED-targets of the structure in order to correspond the field view of the telescope/CCD camera to the dimensions of the captured area and then transform the image pixels in displacement. According to the Eq. 1, it is obvious that for the cases where the monitoring distance is less than $20-30 \mathrm{~m}$ the detected displacement can be up to sub-mm level. 


\subsection{QDaedalus versus RTS}

The main advantage of QDaedalus in structural monitoring versus the Robotic Total Station (RTS), which is broadly used in the last 10 years (Psimoulis and Stiros, 2007; Psimoulis and Stiros, 2012; Psimoulis and Stiros, 2013), is the implementation of the OTR technique instead of the Automatic Target Recognition (ATR) of the RTS. The ATR technique requires the use of prisms for each target (Psimoulis and Stiros, 2007) in order to achieve high-accuracy measurements, which significantly increase the cost of the monitoring project or may even not be feasible in case of inaccessible locations (Bürki et al., 2010). For such a case QDaedalus can be applied, by monitoring several points via LEDs instead of prisms, thus significantly reducing the cost, or even without LEDs in the case of inaccessible targets, where the micro-triangulation technique can be followed (Bürki et al., 2010). Furthermore, the performance of ATR decreases for large distances (>200 m), and decreases for short distances $(<30 \mathrm{~m})$ due to the slightly varying intensity of the laser beam of the RTS (Kirschner and Stempfhuber, 2008).

Additionally the $60 \mathrm{~Hz}$ sampling rate of the CCD camera, or even higher by limiting the image size, and the possibility of synchronizing several QDaedalus systems with the triggering device enables the synchronized estimation of the high-rate displacement time series of several targets of the monitored structure. On the contrary, in RTS monitoring one system per prism is required, while the maximum recording frequency is limited up to $10-20 \mathrm{~Hz}$ with apparent instabilities (Psimoulis and Stiros, 2007; Stiros et al., 2008; Psimoulis and Stiros, 2012). Furthermore the drawback presented by QDaedalus in estimating the 2-D displacement can be alleviated by combining two different QDaedalus (Bürki et al., 2010).

\subsection{QDaedalus versus video-recording systems}

In the recent years, the development of the photogrammetry techniques (i.e. CCD sensors, SLR, video and high-speed camera, etc.) and the powerful computers resulted in creating optical measuring systems of high precision and accuracy (Bösemann, 2005). Several manufacturers, such as AICON (MoveInspect HF), GOM (ATOS Triple Scan), Leica (T-Cam system), etc., created advanced instruments fully automated based on high-resolution cameras and of high rate frames (up to $2000 \mathrm{~Hz}$ ), which are broadly used mainly in industrial applications (Luhnmann, 2010). 
There are different set-ups of cameras in order to make their function more adjustable in the different applications (Bösemann, 2005) and in combination with the developed techniques, it is possible the estimation the 3-D displacement and the orientation of the object (6DOF; Luhmann, 2009; Su and Zhang, 2010). In many applications two or three CCD sensors/cameras are fixed in one portable device on a tripod (e.g. MoveInspect by AICON, HandyScan by GOM), for monitoring the 3-D deformation of an object, e.g. a car (Luhmann, 2010), turbine blade (AICON 3D systems, 2014), etc. with sub-mm accuracy. In other cases the deployment of the sensors is made in a pre-defined network with the control points of the camera-sensors at the perimeter, covering properly the surface of the monitored object (Luhmann, 2010; Schmocker, 2011).

Even though, the above techniques achieve high accuracy with high sampling resolution, they are designed to be used properly for indoor industrial monitoring, short monitoring distances and focusing mainly on specific parts of a structure. Even though, the above techniques can be used for outdoor monitoring, by choosing the appropriate lenses, they are not tested and assessed properly for outdoor conditions. As the QDaedalus is developed for astro-geodetic measurement, it is tested for outdoor conditions. This main advantage is reinforced by the fact that the QDaedalus can be used for the monitoring of the 3-D displacement of outdoor civil engineering structures (i.e. stiff bridges, etc.), by forming a network with two or more QDaedalus systems. The deployment of a network of QDaedalus systems is fast, of high accuracy and easily oriented thanks to the engaged Total Station. Furthermore, an efficiently designed QDaedalus survey network, apart from the 3-D displacement, can restrict also the measurement errors by forming the appropriate triangulation and adjusting the measurements (Bomford, 1980). Additionally, the relative low frames sampling rate with respect the other imagerecording techniques, is not an issue for the majority of the civil engineering structures, where the main modal frequencies are up to $30-40 \mathrm{~Hz}$.

Finally, the main advantage of QDaedalus versus other similar developed techniques combining theodolites with camera (Zhang et al., 2012; Wu and Wang, 2013) is that the CCD sensor is adjusted directly on the telescope of the Total Station making known the exact position and orientation of the captured images. The latter simplifies the technique by avoiding the calibration of the camera with respect to the position of the theodolite/total station and by limiting the potential errors, which may be introduced during the transformations of the measurements in the common coordinate system. 


\section{QDaedalus Monitoring of a Rigid Structure}

In this study, QDaedalus is implemented for the monitoring of the response of the prototype pumppressure "Tensairity" beam, which was excited by impulse forces, caused by hammer hits. The structural response was recorded by the QDaedalus systems and additionally by triaxial lightweight MEMs accelerometers. The reliability and sensitivity of the QDaedalus measurements are evaluated in terms of determination of the modal frequencies. Thus, the assessment of the QDaedalus accuracy was based on the comparison of the QDaedalus modal frequency estimations with the estimations derived by the accelerometers, which are commonly used for the estimation of structures modal frequencies (Moschas and Stiros, 2014). The experiments were carried out for different pressure conditions of the pneumatic beam.

\subsection{Tensairity Beam}

The main concept of the Tensairity beam, which is developed and patented by the Swiss company Airlight Ltd., consists of two elements, a tension and a compression element, connected at the two edges of the beam, while an inflated fabric membrane is positioned between them, in order to stabilize the compression element against buckling (Luchsinger et al., 2004; Luchsinger and Galliot, 2013). The structure is very light, subjected in test vibrations. The vibrations of the beam are controlled, by adjusting the pumped air of the fabric membrane, which basically define the rigidity and the modal frequencies of the beam.

\subsection{Experiments}

The experiment was held on April 30, 2013 in Bauhalle Lab. of ETH Zurich. The length of the beam is $5 \mathrm{~m}$, with two aluminum, curved elements and a membrane element made of polyamide based fabric. The maximum height of the inflated beam is $0.5 \mathrm{~m}$ attained at mid-span (Fig. 2).

There were two target-points for the beam, Target 1 and Target 2, which were monitored both by QDaedalus and triaxial accelerometers located close to the mid- (L/2) and quarter-span (L/4) of the beam, respectively. The two monitored points were not placed exactly on the middle and quarter span in order not to coincide with nodes of the modal shapes of the beam, which could lead to zero displacement for certain modal frequencies. In each monitoring point, a LED was setup and used as 
target for QDaedalus. Each LED was fixed in the front opening of a copper tube in order to avoid potential increase of the LED temperature and was subsequently set in a PVC case. The latter configuration, along with the accelerometer, were fixed on the upper element of the beam, with fixed wooden pads in between, used as the impact surface for the hammer hits.

In addition, eight more points of the upper element were monitored, where triaxial accelerometers were set. These points were not included in our study, as they could not be directly compared with the QDaedalus measurements.

Regarding the accelerometers, they were triaxial lightweight MEMS (STMicroelectronics LIS344ALH) of $\pm 2 \mathrm{~g}$ range, with sensitivity of $3.3 / 5 \mathrm{~V} / \mathrm{g}$ and acceleration density noise $50 \mu \mathrm{g} / \operatorname{sqrt}(\mathrm{Hz})$. The vibration response acceleration signals are sampled at frequency of $1250 \mathrm{~Hz}$. However, the frequency range of interest is $0-30 \mathrm{~Hz}$, so the response signals are filtered and downsampled by a factor of 20 before processed (final sampling period equal to $0.016 \mathrm{~s}$ ).

A total of five experiments were held, corresponding to five different beam pressures $(200,250,300$, 350 and 400 mbar) and resulting to different sets of modal frequencies. Twenty hammer impulses were executed for each pressure experiment, two at each wooden pad with a total duration of each experiment not exceeding the 120 seconds. For the case of the QDaedalus, the measurements were lasting about 180 seconds by recording 30 extra seconds before and after each experiment, for the estimation of the noise level of QDaedalus measurements and potential calibration.

Two QDaedalus systems were used for the monitoring of the two targets; one QDaedalus system for each LED. The two QDaedalus systems were connected with the triggering device in order to simultaneously acquire images of the two LED with the same sampling frequency. The QDaedalus systems were equipped with a TCA1800 Leica Total Station, where the clip-on Guppy F080C digital camera was setup and were adjusted for capturing image of 100x100 pixels per frame with a recording frequency of $57.924 \mathrm{~Hz}$, which was defined by the triggering device. The CCD cameras of both systems were connected with a laptop where their images were recorded. The potential recording frequency was reaching up to $100 \mathrm{~Hz}$ but it was limited by the recording capacity of the laptop.

\section{QDaedalus data processing}

\subsection{Data pre-processing}


For each one of the five experiments and each QDaedalus system a group of $\sim 11000$ images was derived in BMP format. The implemented software Q2DGaussianMatching and the Circle Matching algorithm were used for the extraction of each LED position in pixels. Based on the known triggered sampling frequency and considering no loss of captured images, the time series of the position in pixels of each LED was derived, with respect to the local 2-D arbitrary Cartesian Coordinate System defined by the image field of each QDaedalus system.

Finally, the time series of the position of two LED in pixels were transformed in position time series (in meters) by applying relationship (1) for the corresponding distances of the two QDaedalus systems from the two target 1 and 2 (Fig. 3). For each LED two time series of its position were provided on the $\mathrm{x}$ - and z-axis of the local coordinate system, expressing its displacement on the longitudinal and vertical direction, respectively. While the vertical z-coordinate axis expresses the vertical axis of the beam oscillation, the horizontal $\mathrm{x}$-coordinate axis does not directly the longitudinal beam axis, and further axis transformation is needed. However, only the vertical axis is examined in this study, since this is where the main excitation of the beam was expected.

Furthermore, in the LED time series of the vertical axis, linear trends were observed, corresponding to a potential upward movement of $\sim 0.1 \mathrm{~mm} / 120 \mathrm{sec}$, caused probably by the small amount of continuous air supply during the experiments. These linear trends were removed and the final time series of each LED, expressing the displacement during each experiment is shown in Fig. 4.

\subsection{Estimation of Measurement Noise}

The interval before and after the impulse excitation, which corresponded to a "calm" period corresponds to the noise of QDaedalus measurements. These noise time series were mainly reflecting the nearby disturbances in the lab environment (other experiments in lab, etc.) as well as potential reflection/refraction disturbances of QDaedalus measurements, which however are expected to be rather small within indoor conditions.

From the analysis of noise time series the standard deviation was computed and the corresponding noise level was defined as the 3- $\sigma$ zone. In Fig. 5 a representative noise time series of the vertical component is presented, corresponding to the interval before the experiment \#1 (200mbar airpressure), from where a $3-\sigma$ noise zones of $0.024 \mathrm{~mm}$ and $0.008 \mathrm{~mm}$ were computed for Target 1 and Target 2, respectively. The time series also indicates a periodic pattern with larger amplitude in Target 
1 than in Target 2, corresponding to a frequency of $24.48 \mathrm{~Hz}$. This frequency was detected in all the noise time series of the 5 experiments. Furthermore, the same periodicity was also detected in other outdoor QDaedalus experiments of different environmental conditions corresponding to a "calm" period. This frequency corresponds to an amplitude of 10-20 $\mu \mathrm{m}$ and probably expresses noise caused by the experimental setup i.e. the copper tube set in a PVC case, which might not be totally fixed.

In all the noise time series of the 5 experiments the amplitude in Target 1 is larger than that of Target 2 appearing the same periodicity, which indicates that the noise which is caused by disturbances of other experiments in the laboratory is potentially filtered through the modal response of the beam i.e. larger noise level in the mid-span than in the quarter-span.

Finally, the highest $3-\sigma$ noise zone was $0.027 \mathrm{~mm}$, corresponding to Target 1 for the $300 \mathrm{mbar}$ pressure and proved to be significantly smaller than the expected oscillation signal, without affecting the oscillation time series analysis. The latter noise zone was used for the definition of the oscillation time series of the two targets.

\subsection{Analysis of the oscillation time series}

The oscillation signal corresponding to the impulse response was clearly distinguished in the vertical time series of both targets for all experiments. In Fig. 4 is presented the vertical time series of the two targets corresponding to the air-pressure $200 \mathrm{mbar}$, where the oscillation signal of each one of the 20 impulses is clearly distinguished as it exceeds the noise level of $0.027 \mathrm{~mm}$. The different peak amplitude of the impulse oscillations for each hit, are due to the different points of the excitations and the variance of the force of the hammer hits.

The relatively different response of the two targets in amplitude, due to the differences of the modal shapes at the position of each target is also evident from the time series. The vertical oscillation of Target 1 and Target 2, for hit excitation at Target 2 and 1 and for air-pressure of 200 mbar, are presented in Fig. 6. The amplitude of the displacement is larger in Target 2 than in Target 1, when the hit is at Target 2, with peak displacement of $\sim 0.5 \mathrm{~mm}$ and $\sim 0.3 \mathrm{~mm}$, respectively. The response of the two targets is reversed for a hit at Target 1 , as the peak displacement of Target 2 and 1 are $0.2 \mathrm{~mm}$ and 0.8 , respectively. However, the relative difference of the displacement of the two targets can be checked only qualitatively, as the poor resolution of the QDaedalus measurements relatively to the high-frequency motion of the beam, results to mis-recording some peaks of displacement-cycles. 
The loss of peak displacement can be observed by comparing the displacement time series of the QDaedalus and the one computed by the corresponding accelerometer time series (Fig. 7). The acceleration time series was integrated twice and filtered using a Chebysev high-pass filter in order to remove the low frequencies $(<1 \mathrm{~Hz})$ and avoid the drift in the displacement time series (Stiros, 2008). With respect that the accelerometers and QDaedalus systems were not synchronized and by comparing the two displacement time series is clear that both of them express displacement of similar amplitude, with the QDaedalus missing some of the peaks of displacement-cycles though. However, the displacement time series of the accelerometer might still lose displacement information through the high-pass filtering procedure. Thus, the methodology of integrating the acceleration time series to displacement was not followed and the assessment of the QDaedalus records was based on the comparison of the estimated modal frequencies with those from the accelerometers measurements.

For the estimation of the modal frequencies, the Discrete Fourier Transform (DFT) was used and from the derived spectra, the most significant peaks were detected according to their amplitude. The resolution precision of the DFT for the QDaedalus time series was rather small $(\sim 0.37 \mathrm{~Hz})$ due to the relative short duration of the excitation, which did not exceed 1.5 sec. In Fig. 6 are presented the spectra of the DFT analysis of the corresponding time series of QDaedalus and accelerometer records. Even though significant frequencies even up to $100 \mathrm{~Hz}$ were estimated in the accelerometers records, the frequency range was limited up to $30 \mathrm{~Hz}$ for comparison with the QDaedalus estimations.

The main modal frequency of $\sim 27.4 \mathrm{~Hz}$ is estimated by both accelerometer and QDaedalus, for hits at Target 1 and 2. Also the spectra of the acceleration and displacement appear of a similar pattern, retaining the relative amplitude of the peaks between the two targets, thus indicating the consistency between the time series of the two measuring techniques. Moreover, there are frequencies in the lower range depicted in the QDaedalus spectra $(\sim 5 \mathrm{~Hz})$ which do not appear in the accelerometer spectra.

The DFT analysis of the accelerometer and QDaedalus time series was done for all the examined airpressures. In Figure 8 the time series and the corresponding spectra of the accelerometer and QDaedalus records are presented for Target 1, for impulse hit at the same point. The time series are again of $\sim 1.5 \mathrm{sec}$ duration. From the peaks of the spectra it is obvious that the two main estimated modal frequencies for the low air-pressure (200 and 250mbar) are consistently estimated both by accelerometer and QDaedalus (Table 1), considering also the QDaedalus spectra resolution $(\sim 0.4 \mathrm{~Hz})$ and the limitation of the Nyquist frequency of the QDaedalus spectra $(\sim 28.96 \mathrm{~Hz})$. By increasing the 
air-pressure the beam becomes more rigid and the modal frequencies are shifted to higher values. This is made rather clear from the acceleration spectra, as the increase of the air-pressure lead to increase of the modal frequencies values. Specifically for air-pressure higher than $300 \mathrm{mbar}$ one of the two main modal frequencies is above the range of the $30 \mathrm{~Hz}$ of the spectra. Regarding the QDaedalus spectra, the frequency shift to a higher value is also detectable with increase of the air-pressure, even for the case of high air-pressure $(23.78 \mathrm{~Hz}$ for $350 \mathrm{mbar}$ and $24.61 \mathrm{~Hz}$ for $400 \mathrm{mbar})$. The only exception is the case of 300mbar air-pressure, where the inconsistency of the detected frequency might be again a result of the relative poor resolution of the QDaedalus spectra.

\section{Discussion}

The comparison of the QDaedalus oscillation time series clearly shows the qualitative difference of the corresponding waveforms due to the different contribution of the modal shapes and the relative position of the excitation point (Fig. 6b). The latter is also obvious in the corresponding spectra, where the same frequencies are mainly detected (Fig. 6d). However, there are also cases, where the contribution of some modes is null for one of the two targets and the corresponding oscillation is weak. The latter leads to detection of a frequency peak only in one of the two targets; representative is the case of the excitation at Target 1 and air-pressure 200 mbar, where the frequencies of $\sim 23.06$ and 17.14 Hz are detected only in Target 1 (Fig. 6h). This effect is probably amplified by the relative poor resolution of the QDaedalus records (Fig 6f).

Furthermore, the estimation of the modal frequencies by QDaedalus records is rather consistent with that of the accelerometer records, especially for the lower air-pressure (200 and $250 \mathrm{mbar}$ ), which is also evident from the similarity of corresponding spectra-form for both of the two targets (Fig. 6c,d). Potential differences (up to $0.3-0.4 \mathrm{~Hz}$ ) between the detected frequencies of the accelerometer and QDaedalus spectra are mainly the result of the poor precision resolution of the QDaedalus spectrum (i.e. $\sim 0.37 \mathrm{~Hz}$ ). However, from the QDaedalus spectra there are also observed relative low frequencies e.g. $\sim 5 \mathrm{~Hz}$ and $17-18 \mathrm{~Hz}$; the frequency of $5 \mathrm{~Hz}$ corresponds to very low amplitude expressing mainly noise, while the frequency of $17-18 \mathrm{~Hz}$ might correspond potentially to torsional or other mode, as it is also detected in acceleration spectra but not regarding the vertical component. 
The increase of the air-pressure resulting to the increase of the beam rigidity and the increase of the modal frequencies values is also detectable in the QDaedalus measurements. The latter is made clear by the corresponding spectra where the values of the main detected modal frequencies are increased following similarly the trend of the estimated frequencies by the accelerometer. Though, the increase of the values of the modal frequencies is not so clearly defined by the QDaedalus spectra, mainly for air-pressure higher than 300 mbar, due to i) the relative poor resolution, which leads to a not so precise record of the oscillation waveform and missing peaks of displacement-cycles, ultimately affecting the estimated frequencies, an effect which is observed in Robotic Total Station monitoring (Psimoulis and Stiros, 2008) and ii) the weaker signal of the remained frequencies leading to their less accurate detection.

However, it was possible the estimation of the displacement of the beam in an independent coordinate system, without losing any displacement information, through potential filtering of data (i.e. doubleintegration of the acceleration time series). The advantage of the QDaedalus system was also the potential of monitoring the absolute position of the structure if it was needed by using an appropriate survey network.

\section{Conclusion}

This study was the first attempt for introducing QDaedalus, an implemented technique combining a Total Station with a CCD camera, in structural monitoring. QDaedalus was used for the monitoring of a very rigid structure with only two main modal frequencies below $30 \mathrm{~Hz}$, which was the highest detectable frequency by QDaedalus and oscillation amplitudes not exceeding 0.7-0.8 mm. Based on the experiments, the QDaedalus measurements were precise (noise below $<0.03 \mathrm{~mm}$ ), accurate enough in estimating the modal frequencies below $30 \mathrm{~Hz}$ and also adequately sensitive in detecting the modal frequency changes due to the variation of the beam air-pressure.

Thus, QDaedalus proves to be accurate for the monitoring of rigid dynamic response of civil engineering structure. Further improvement of the QDaedalus can be achieved by increasing the recording frequency, which would refine the displacement time series, the spectra resolution and the frequency detection, provided the major requirement, which is the appropriate monitoring distance $(<30 \mathrm{~m})$, is fulfilled so as to achieve sub-mm accuracy. However, the restriction of the monitoring distance can be limited by upgrading the CCD camera to higher resolution. Finally, the QDaedalus 
monitoring has recently been improved, as it can provide the structure displacement time series in realtime, by using an appropriate laptop, in terms of memory and processor speed, according to the required image resolution and sampling-rate. The potential of using Robotic Total Station (RTS) instead common Total Station, could broaden the application field and improve the applicability and the accuracy of QDaedalus by monitoring movement outside the field of view of the Total Station and hence reducing the monitoring distance.

\section{Acknowledgment}

The preparation of the LED set up was provided P. Sorber, technician of Institute of Geodesy and Photogrammetry of ETH Zurich, and his help is very much appreciated. The article has greatly benefited from the constructive comments of three anonymous reviewers.

\section{References}

AICON 3D Systems (2014) MoveInspect HF: Rotor blade testing at the IWES, Application report, available on: http://aicon3d.com/applications/portable-coordinate-measuring-technique/dynamicmeasurement/iwes.html

Bardakis VG, Fardis MN (2011) A displacement-based seismic design procedure for concrete bridges having deck integral with the piers, Bull. Earthq. Eng., 9(2), 537-560

Behr AJ, Hudnut WK (1998) Monitoring structural deformation at Pacoima dam, California using GPS, Seism. Res. Lett., 299-308

Bomford G (1980) Geodesy, Oxford: Clarendon Press, Ed. $4^{\text {th }}$.

Bösemann W (2005) Advances in photogrammetric measurement solutions, Computers in Industry, $56,886-893$

Breuer P, Chmielewski T, Gorski P, Konopka E, Tarczynski I (2008) The Stuttgart TV towerdisplacement of the top caused by the effects of sun and wind, Eng. Str., 30, 2771-2781

Bürki B, Guillaume S, Sorber P, Oesch H-P (2010) Daedalus: A versatile usable digital clip-on measuring system for Total Stations, Proc. of Int. Conf. on Indoor Positioning and Indoor Navigation (IPIN), 15-17, September, 2010, Zurich, Switzerland 
Chatzi EN, Fuggini C (2012) Structural identification of a super-tall tower by GPS and accelerometer data fusion using a multi-Kalman filter, Proc. of $3^{\text {rd }}$ Int. Symposium on Life-Cycle Civil Engineering IALCCE, 144-151

Chatzi EN, Smyth AW (2013) Particle filter scheme with mutation for the estimation of time-invariant parameters in structural health monitoring applications, Str. Con. Heal. Mon., 20(7), 1081-1095

Fukuda Y, Feng MQ, Shinozuka M (2010) Cost-effective vision-based system for monitoring dynamic response of civil engineering structures, Str. Con. Heal. Mon., 17, 918-936.

Gentile C, Bernardini G (2010) An interferometric radar for non-contact measurement of deflections on civil engineering structures: laboratory and full-scales tests”, Str. Infr. Eng., 6(5), 521-534

Gentile C (2010) Deflection measurement on vibrating stay cables by non-contact microwave interferometer, NDT and E International, 43(3), 231-240.

Hamsphire TA, Adeli H (2000) Moniotoring the behavior of steel structures using distributed optical fiber sensors, J Constr St Res, 53(3), 267-281

Kim DE, Chen, TH, (2012), A virtual erection simulation system for a steel structure based on 3D measurement data, J Mar Sci Appl, 11, 52-58

Koo KY, Brownjohn JMW, Cole R, List DI (2012), Structural health monitoring of the Tamar Bridge, Str Cont Heal Mon, 20(4), 609-625

Kirschner H, Stempfhuber W, (2008) The kinematic potential of modern tracking Total Stations - A state of the art report on the Leica TPS1200+, Proc of $1^{\text {st }}$ Int. Conf on Mach Contr \& Guid, 51-60

Li X, Ge I, Ambikairajah E, Rizos C, Tamura Y, Yoshida A (2006) Full-scale structural monitoring using an integrated GPS and accelerometer system, GPS Sol, 10(4), 233-247

Luchsinger RH, Pedretti A, Steingruber P, Pedretti M, (2004) The new structural concept Tensairity: Basic principles, A. Zingoni (ed.), Progress in Structural Engineering, Mechanics and Computation, A.A. Balkema Publishers, London, 323-328.

Luchsinger RH, Galliot C, (2013) Structural behavior of symmetric spindle-shaped tensairity girders, J Struct Eng, 139(2), 169-179.

Luhmann T, (2009) Precision potential of photogrammetric 6DOF pose estimation with a single camera, ISPRS J Photogr. Rem. Sens., 64, 275-284

Luhmann T, (2010) Close range photogrammetry for industrial applications, ISPRS J Photogr. Rem. Sens., 65, 558-569 
Meng X, Dodson AH, Roberts GW (2007) Detecting bridge dynamics with GPS and triaxial accelerometers, Eng Str, 29(11), 3178-3184

Moschas F, Psimoulis P, Stiros S (2013) GPS-RTS data fusion to overcome signal deficiencies in certain bridge dynamic monitoring projects, Sm Str Sys, 12(3-4), 251-269.

Moschas F, Stiros S (2014) Three-dimensional dynamic deflections and natural frequencies of a stiff footbridge based on measurements of collocated sensors, Str Con Heal Mon, 21(1), 23-42.

Niemeier W, Riedel B, Fraser C, Neuss H, Stratmann R, Ziem E (2008) New digital crack monitoring system for measuring and documentation of width of cracks in concrete structures, Proc. of $13^{\text {th }}$ FIG Symp. on Deformation Measurement and Analysis and $14^{\text {th }}$ IAG Symp. on Geodesy for Geotechnical and Structural Engineering, Lisbon, 12-15 May, 2008.

Olaszek P (1999) Investigation of the dynamic characteristic of bridge structures using a computer vision method, Meas., 25, 227-236

Psimoulis P, Stiros S (2007) Measurement of deflections and oscillation frequency of engineering structures using robotic theodolites (RTS), Eng Str, 29(12), 3312-3324.

Psimoulis P, Stiros S (2008), Experimental assessment of the accuracy of GPS and RTS for the determination of the parameters of oscillation of major structures, Comp-Aid Civ Infr Eng, 23(5), 389-403.

Psimoulis P, Stiros S (2012) A supervised learning computer-based algorithm to derive the amplitude of oscillations of structures using noisy GPS and robotic theodolites (RTS) records, Comp. Str., 9293, 337-348.

Psimoulis P, Stiros S (2013) Measuring deflections of a short-span railway bridge using Robotic Total Station (RTS), J Br Eng, 18(2), 182-185.

Roberts GW, Meng X, Dodson A (2004), Integrating a global positioning system and accelerometers to monitor deflections of bridge, J. Surv. Eng., ASCE, 65-72

Spiridonakos M, Fassois S (2009) Parametric identification of a time-varying structure based on vector vibration response measurements, Mech. Sys. Sign. Proc., 23(6), 2029-2048

Stiros S, Psimoulis P, Kokkinou E (2008) Errors introduced by fluctuations in the sampling rate of automatically recording instruments: experimental and theoretical approach, J. Surv. Eng., ASCE, 134(3), 89-93 
Stiros S (2008) Errors in velocities and displacements deduced from accelerographs: An approach based on the theory of error propagation, S. Dyn. Earthq. Eng., 28, 415-420

Stiros, S., Psimoulis, P. (2012) Response of a historical short-span railway bridge to passing trains: 3D deflections and dominant frequencies derived from Robotic Total Station measurements, Eng. Str., $45,362-371$

Su Z, Zhang Q (2010) Dynamic 3-D shape measurement method: A review, Optics and Laser in Engineering, 48, 191-204

Wu B, Wang B (2013) Automatic measurement in large-scale space with the laser theodolite and vision guidying technology, Adv Mech Eng,

Zarikas V, Gikas V, Kitsos CP (2010) Evaluation of the optimal design "cosinor model" for enhancing the potential of robotic theodolite kinematic observations, Meas, 43(10), 1416-1424

Zhang X, Zhu Z, Yuan Y, Li L, Sun X, Yu Q, Ou J, (2012) A universal and flexible theodolite-camera system for making accurate measurements over large volumes, Opt Las in Eng, 50, 1611-1620 


\section{Figure Captions}

Figure 1: a) Sketch of the monitoring of a beam using QDaedalus. A CCD camera, clipped on the total station, captures the position of the LED (red circle) on the beam. The LED position in pixels (Px, Py) in 2-D local coordinate system derived from the image processing of QDaedalus images. b) Sketch of the monitoring of a beam using two QDaedalus systems in different control points. The different angular view provide the 3-D displacement of the LED-target with respect the beam Cartesian coordinate system $\left(\mathrm{x}_{\mathrm{g}}, \mathrm{y}_{\mathrm{g}}, \mathrm{z}_{\mathrm{g}}\right)$.

Figure 2: The tensairity beam with the two monitored points approximately in the midspan (Target 1) and at the quarter-span (Target 2). At each monitored point is set up the accelerometer, the wooden pad and the LED in the PVC case.

Figure 3: The set up of the two QDaedalus systems for the monitoring of the beam. Each QDaedalus system monitor one LED target from approximately the same monitoring distance (5.6 and $5.8 \mathrm{~m}$, respectively).

Figure 4: Time series of the vertical component of QDaedalus measurements for the experiment of $200 \mathrm{mbar}$ air-pressure. It is obvious the calm period of 40 and 30 seconds before and after the hammer impulses, and the 20 hammer impulses excitations during the experiment.

Figure 5: (top) The QDaedalus time series before the impulses of experiment 1, expressing the measurement noise of the monitored points (Target 1, Target 2). (bottom) The DFT analysis reveals a periodic signal of $24.48 \mathrm{~Hz}$, corresponding to the noise of the QDaedalus measurements

Figure 6: Representative acceleration $(\mathrm{a}, \mathrm{e})$ and displacement $(\mathrm{b}, \mathrm{f})$ time series of the two targets as they derived from the accelerometer and QDaedalus records, for hit over Target 2 (top) and Target 1 (bottom), and the corresponding spectra based on the DFT analysis (c,d,g,h). The air-pressure of the tensairity beam is $200 \mathrm{mbar}$. 
Figure 7: The displacement time series of Target 2 (a) and 1 (b), as they derived from the QDaedalus records and the corresponding accelerometers records after the appropriate double integration and filtering. The beam air-pressure is 200 mbar and the pulse hit was executed on Target 2.

Figure 8: The time series (left) and the corresponding spectra (right) of the accelerometer and the QDaedalus system of the Target 1 for hits on the same point for beam air-pressure 200, 250, 300, 350 and 400mbar.

\section{Table Caption}

Table 1: The dominant estimated frequencies of the accelerometer and QDaedalus system as they are extracted by the spectra of Fig. 8 

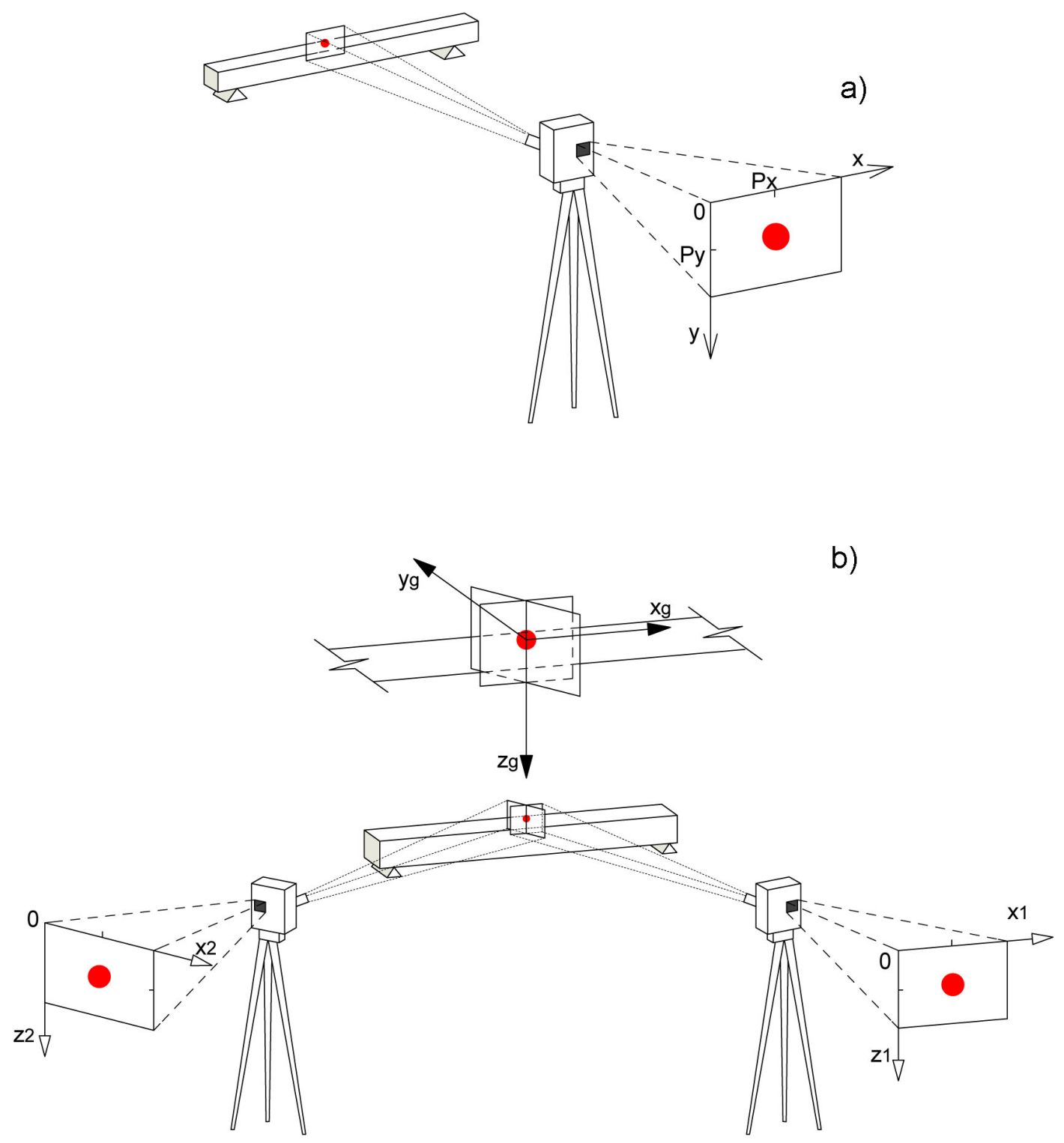

Figure 1 


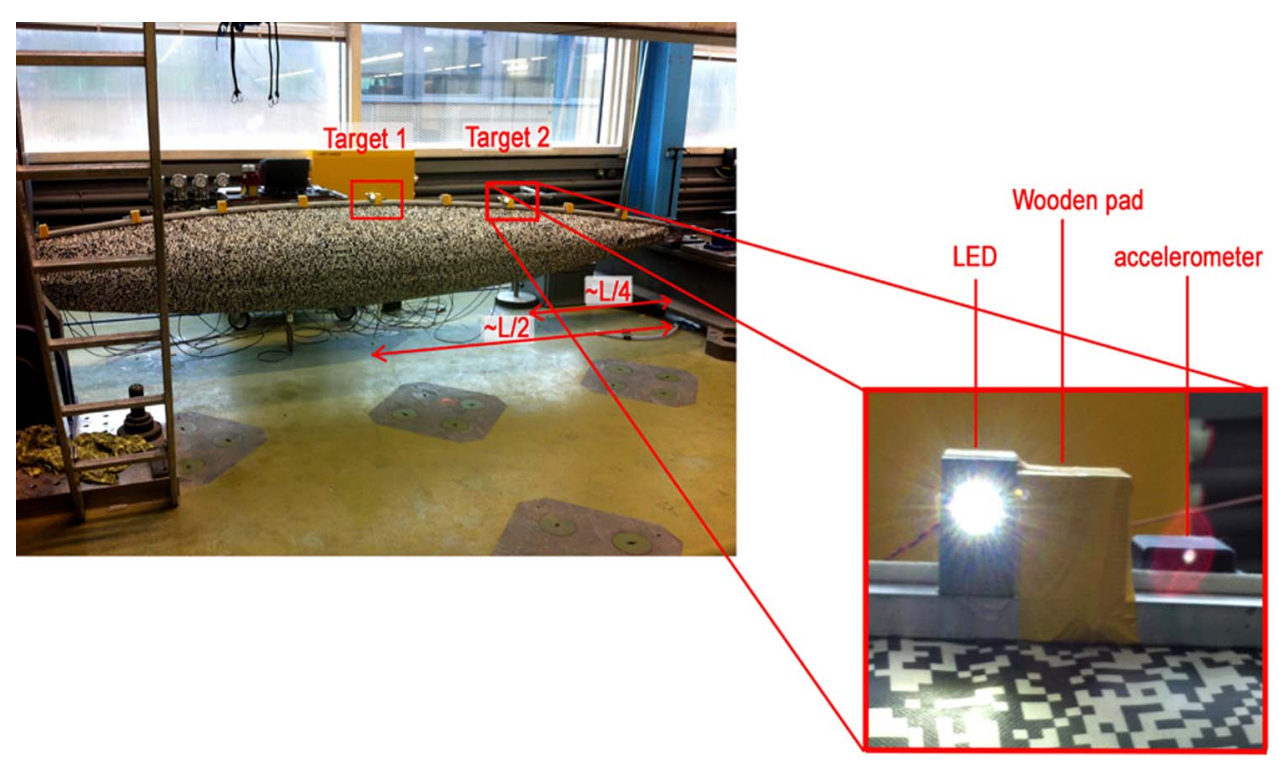

Figure 2 


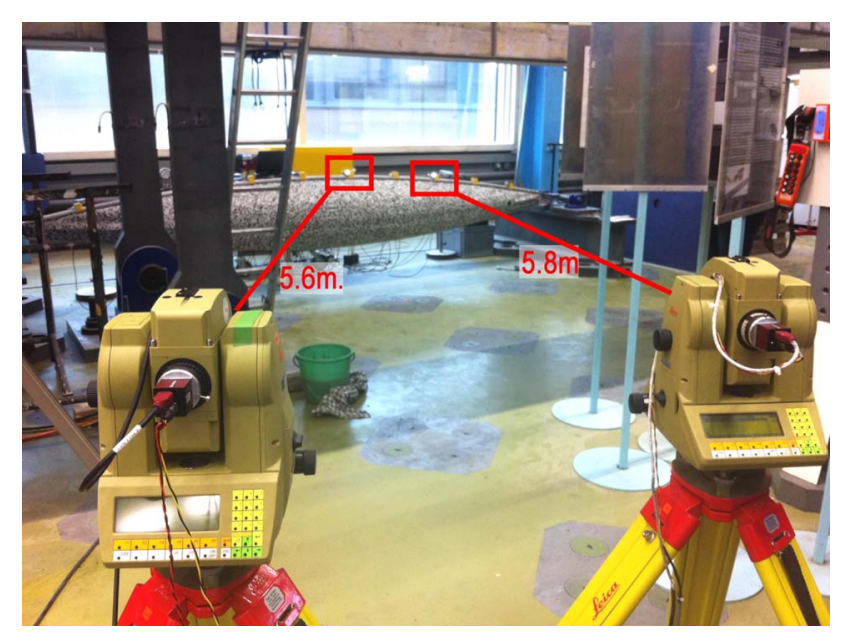

Figure 3 


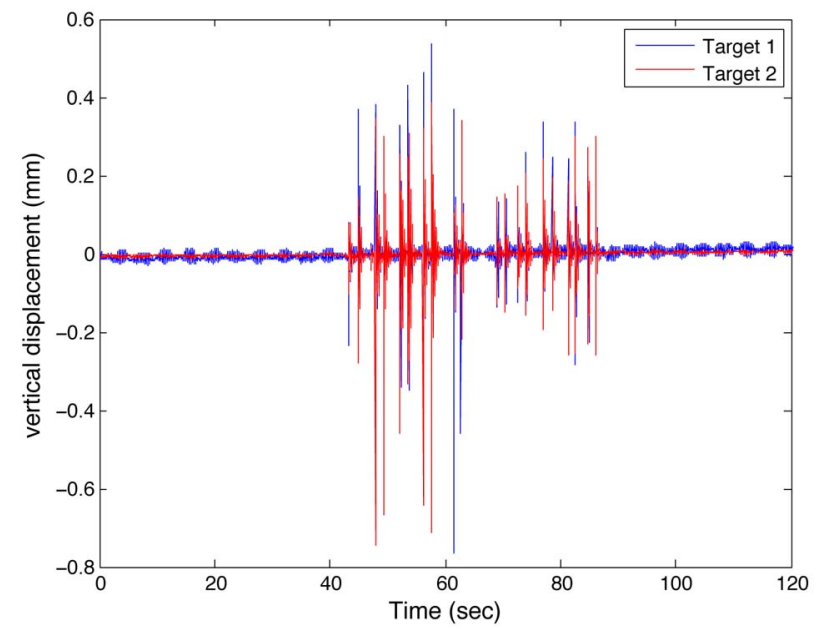

Figure 4 

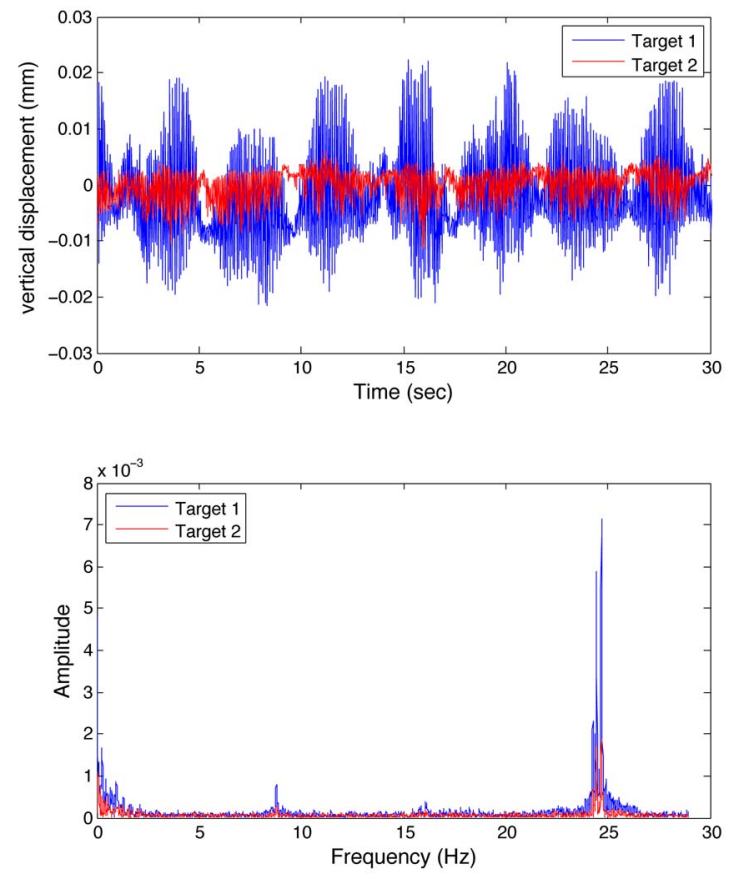

Figure 5 

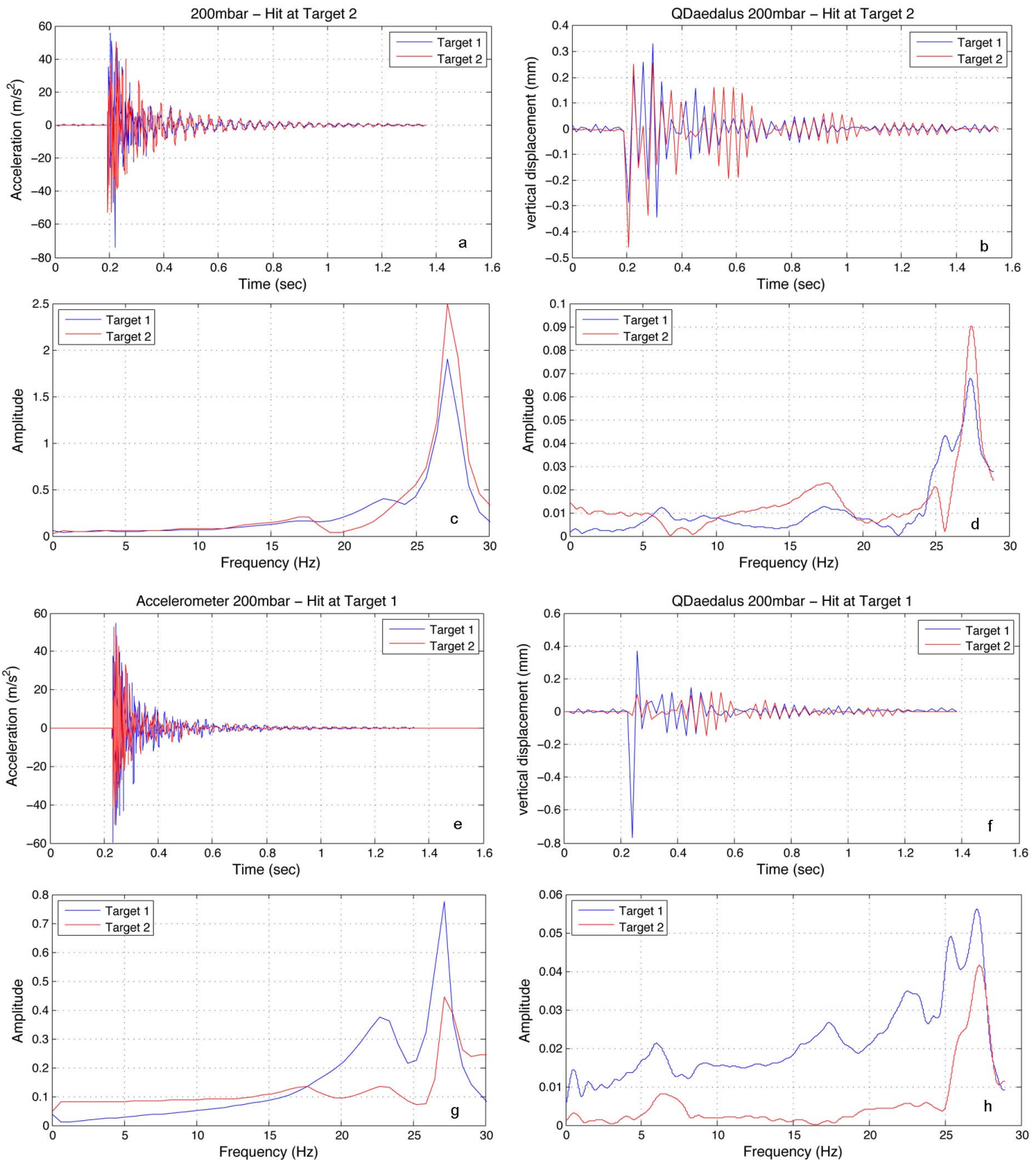

Figure 6 

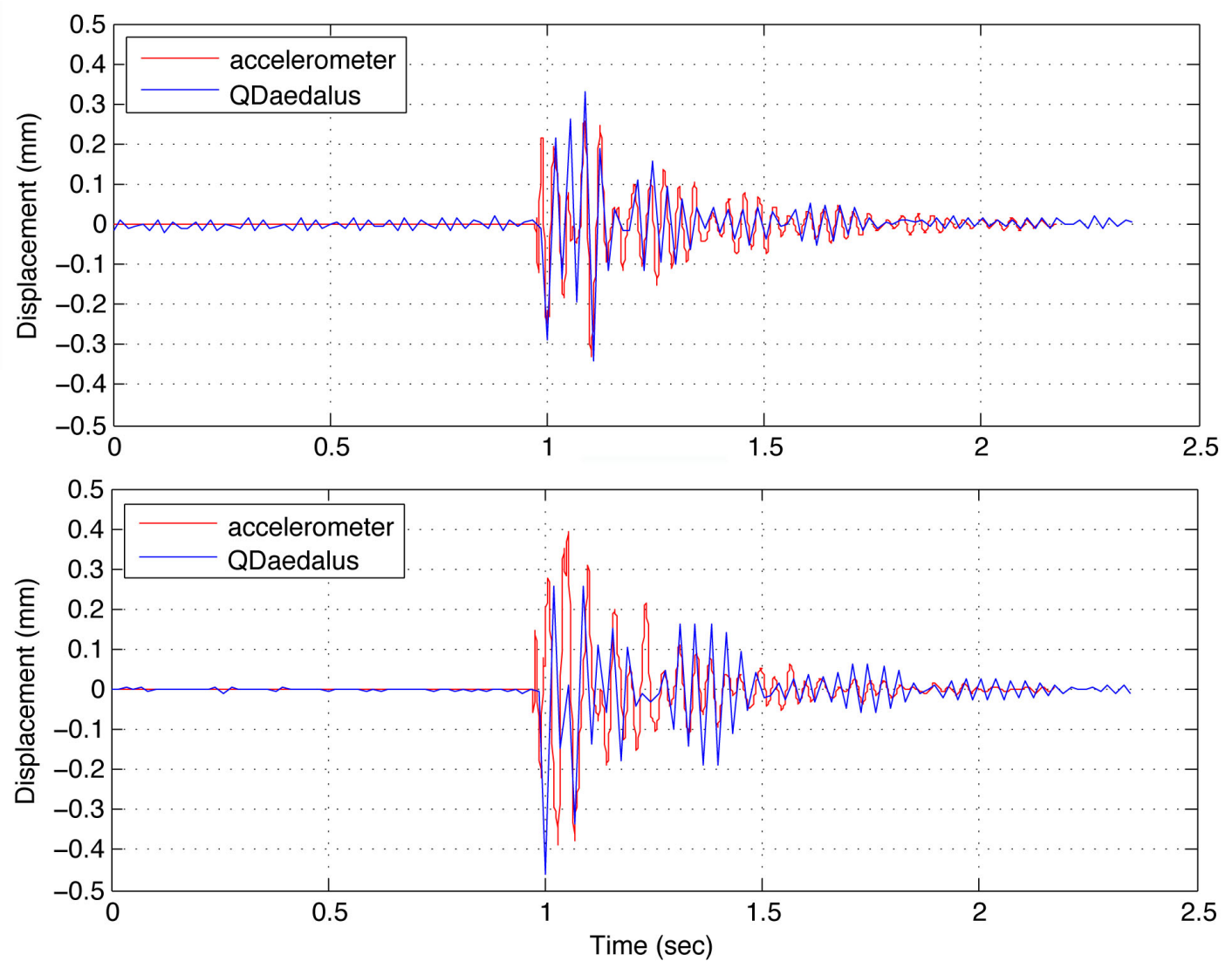

Figure 7 

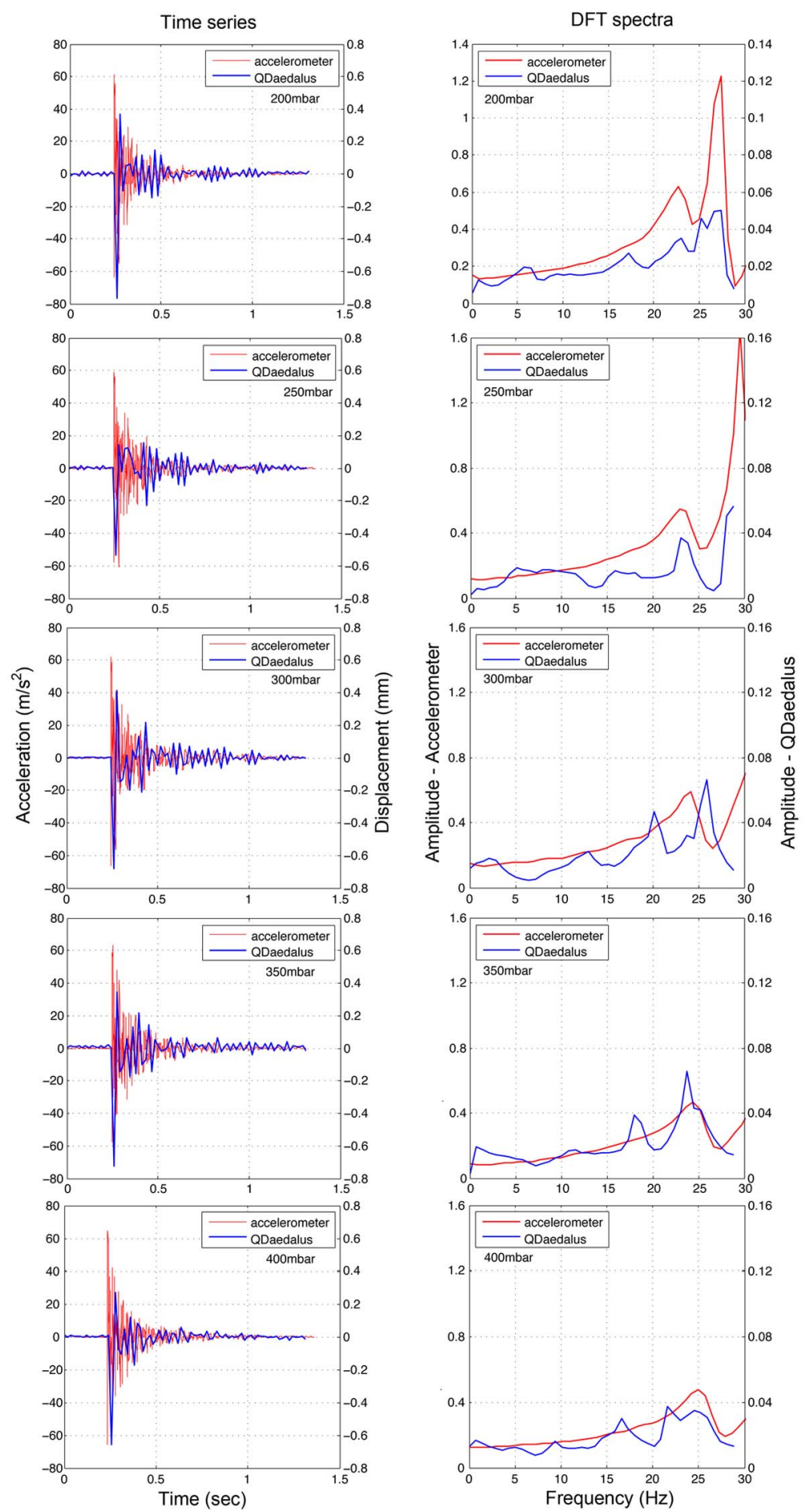

Figure 8 
Table 1

\begin{tabular}{cccccc}
\hline \multirow{3}{*}{ Pressure (mbar) } & \multicolumn{5}{c}{ Frequency $(\mathrm{Hz})$} \\
\cline { 2 - 6 } & \multicolumn{2}{c}{ Accelerometer } & \multicolumn{3}{c}{ QDaedalus } \\
\cline { 2 - 6 } & 1st & 2nd & 1st & 2nd & 3rd \\
\hline \hline 200 & 27.41 & 22.71 & 27.39 & 25.23 & 23.06 \\
250 & 29.57 & 22.92 & 28.83 & 23.06 & - \\
300 & 24.20 & - & 25.95 & 20.18 & 23.78 \\
350 & 24.33 & - & 23.78 & 25.23 & 18.02 \\
400 & 24.97 & - & 21.62 & 24.61 & 16.58 \\
\hline
\end{tabular}

